\title{
Electron Diffuse Scattering Study of Perovskite Thin Films
}

\author{
J. Pacaud,* F. Pailloux,* \\ * Lab de Physique des Matériaux, Université de Poitiers, SP2MI/BP30179, Ave P et M Curie, 86962 \\ Chasseneuil Cedex, France
}

The main requirement for many devices is the growth of a high purity and high structural quality thin film. The perovskite structure is extremely sensitive to the deposition condition and particularly to the temperature and the partial pressure of oxygen. Changes in deposition conditions may lead to a large deviation of the dielectric properties of thin films from those of bulk materials. The chemistry of defects is often proposed as an explanation of this deviation. Beside the oxygen deficiency, the cation stoichiometry seems to play a major role on the structure and properties of the grown film as it might induce the nucleation of structural defects (dislocation loops, stacking fault, RuddlesdenPopper faults...).

Another important parameter for thin perovskite films is the geometrical constraint imposed by the substrate. Most of the time, perovskite exhibit excellent epitaxy on each other and the films are tied to the substrate so the in-plane parameters are not free to reach their bulk equilibrium values. For materials as sensitive to phase transition as perovskite this effect and the associated relaxation processes can be extremely important for the fine tuning of the physical properties of the film.

For perfect crystal, diffuse scattering is mostly inelastic due to phonons, plasmons and other processes. Thermal diffuse scattering or phonon scattering can lead to large effects in the electron diffraction pattern especially near second order phase transition where phonon softening occurs. Additionally, elastic diffuse scattering comes from structural deviations from a periodic lattice [1]. These structural deviations can be defects, partial ordering of otherwise disordered structure or structural fluctuations. By studying diffuse scattering we can obtain information about the crystal imperfections and dynamics which can not be obtained from other characterization methods.

The goal of this study is to characterize, through diffuse scattering in electron diffraction, the structure of defects in the epitaxial layers of perovskite structure and more specifically its influence on the dynamic of the lattice (lattice vibrations, structural fluctuations and continuous phase transition due to phonon softening) [3]. These properties have to be linked with the anomaly of the ferroelectric properties of such thin films. Different strain states can be probed by changing the substrate material or introducing different buffer layers.

The results of STO films deposited on different substrate show different behavior. On STO substrate, at $135 \mathrm{~K}$, the superstructure spots in the thin film, have already the same FWHM than the fundamental reflections, indicating a complete phase transition. This shift toward higher transition temperature in thin STO films has already been observed [4]. On $\mathrm{LaAlO}_{3}$ (LAO) substrate, fine peaks appear near $140 \mathrm{~K}$ in the diffraction pattern of the film revealing a strain induced phase transition.

[1] Herranz G., Basletic M., Bibes, M., Ranchal R., Hamzic A., Tafra E., Bouzehouane K., Jacquet E., Contour J.P., Barthélémy A., Fert A., Phys. Rev. B, 73(2006) p. 064403

[2] Krivoglaz M.A., "Diffuse scattering of X-Rays and Neutrons by Fluctuations", Springer Berlin (1994)

[3] Wang R., Zhu Y., Shapiro S.M., Phys. Rev. B, 61,(2000) p. 8814 
[4] He F, Wells B.O., Shapiro S.M., v Zimmermann M., Clark A., Xi X.X., Appl. Phys. Lett., 83, $123(2003)$

[5] The authors acknowledge Dr K. Bouzehouane from UMR CNRS/Thalès, Palaiseau, France, for providing the samples.

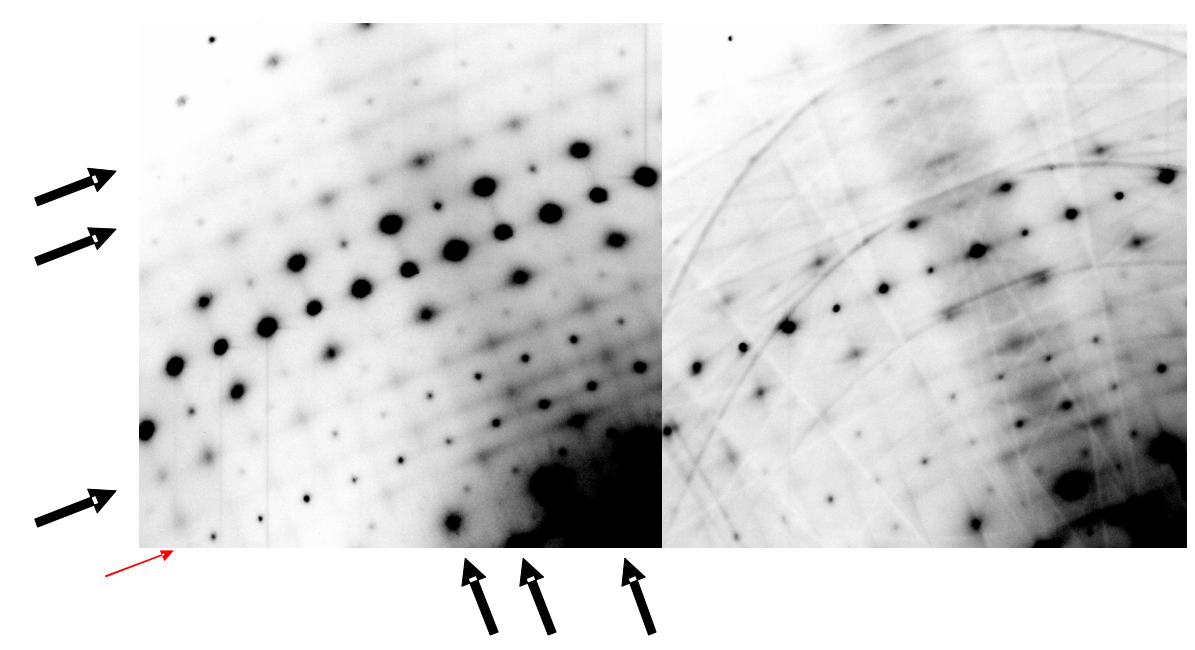

FIG. 1. Diffraction patterns along the [061] zone axis for STO film (left) on STO substrate (right). The transmitted beam is out of the picture below the right corner. Black arrows show the sheets of diffuse scattering in the $\langle 100\rangle *$ planes. Red arrow shows the row of low temperature phase spots and the diffuse streak in the [100]* direction.

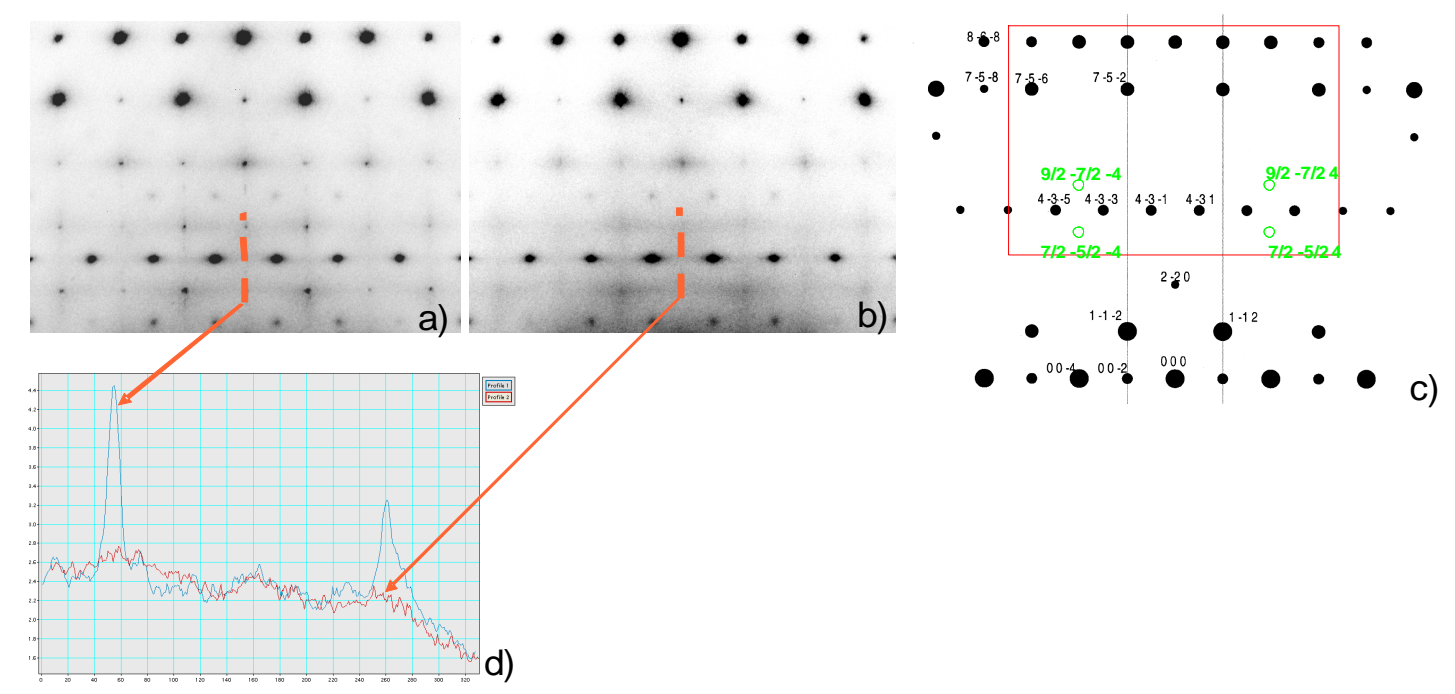

FIG. 2. Diffraction pattern of STO on LAO substrate $0.4^{\circ}$ off the [710] zone axis. a) $130 \mathrm{~K}$ diffraction pattern. b) after one hour of observation (temperature slightly rised). c) kinematical simulation of the I $4 / \mathrm{mcm} 105 \mathrm{~K}$ low temperature phase. d) Intensity profile along the orange broken lines in a) and b) 